influence of the Thomson effect, variation of surface tension with temperature and surface purity, and also the influence of electrodynamic forces were discussed; internal circulating motion in the liquid and modification of the shape of the bridge will be produced. A possible theoretical explanation was proposed for the reported breaking of bridges below the boiling voltage under certain conditions.

The formation and properties of molten metal bridges was discussed by R. H. Jones (Physics Department, University College of Swansea), who analysed the various theories which have been advanced to account for bridge transfer, paying particular attention to temperature distribution in a contact. He described experimental work on the pip and crater formed in a single contact operation, and showed slides illustrating in detail the growth and rupture of the bridge both with single-metal and bi-metallic contacts. Mr. Jones considers that his results can be explained on the basis of the Thomson effect for single-metal contacts and by also taking into account the differing thermal and electrical properties of the metals in a bi-metallic contact.

Prof. F. Llewellyn Jones (Physics Department, University College of Swansea) spoke on the erosion produced by electrical discharges and analysed the basic processes occurring at both the 'make' and the 'break' phases of contact operation. He emphasized the hitherto unsuspected importance of low-voltage discharge phenomena which can occur between electrodes just about to meet $\left(\sim 10^{-4} \mathrm{~cm}\right.$. apart). The problem consists of finding the source and emission mechanisms of the electrons which initiate the discharge and also the means by which the electron current is amplified to produce a discharge avalanche. He reported experiments, carried out with E. T. de la Perrelle and G. G. Morgan, in which the rate of electron emission from cold metal surfaces was investigated by using a pulse technique in which the production of a single electron could be recorded. Comparatively high electron emission from contact surfaces was obtained with electric fields as low as $10^{4} \mathrm{~V} . / \mathrm{cm}$, and the variation of the emission with electric intensity showed that the mechanism was field emission. The emission depended very greatly on the nature of the electrode surface; for example, a thin oxide tarnish layer enhanced the emission at least a thousand times, while the presence of dust (due to the condensation of metal evaporated by the microscopic discharges) could further enhance the emission by a similar factor. Thus the probability of initiation of discharge during the operation of a pair of contacts greatly depends on the surface state of those contacts, and it might be expected that erosion would be greatly increased when those surfaces are dusty or covered with thin tarnish layers. Removal of such layers practically eliminates the discharges and the erosion, while the sweeping of positive ions to the layers greatly enhances the ernission.

In distinguishing between two types of films on metal surfaces, namely, tarnish films and grease films, Dr. J. C. Chaston (Johnson Matthey and Co., Ltd.) discussed the tarnishing of copper: in pure air (containing no sulphur) copper, although apparently clean, is covered by an oxide film $85 \mathrm{~A}$. thick which prevents further oxidation. The atmosphere, which both in town and country contains traces of hydrogen sulphide, produces a film of oxide and sulphide on copper about $450 \mathrm{~A}$. thick and reddishbrown in colour; but the thin oxide film, produced in pure air, will protect against the second. Properties of other contact metals were described. He raised a number of questions on the actual area of contact of two apparently plane surfaces, the welding of clean metal surfaces, the continuity or otherwise of the surface film at contact spots, the sticking of contacts, and the passage of current through films $20 \mathrm{~A}$. thick. D. O. Walter (Johnson Matthey and Co., Ltd.) dealt mainly with practical considerations in the design of light-duty sliding contacts, and he also discussed the properties of electrodeposited rhodium. Rhodium $(<0.001$ in. $)$ is often deposited on a silver substrate 0.0005 in. thick, and provides a useful contact surface, but the contact pressure must not be so high as to deform the silver substrate plastically. C. W. A. Mitchell and T. F. A. Urben (Telephone Branch, Post Office Engineering Department) gave an account of the exacting requirements of contact devices in telephone engineering, and discussed the use of various materials under a variety of mechanical conditions. Silver is generally used in low-voltage contacts : a ciné-film showed how contact bounce can be eliminated (or reduced) by proper mechanical design.

These papers were followed by discussion devoted mainly to the influence of tamish films; from this it may be concluded that light-duty contact operation is greatly determined by the nature of the contact surface, because that surface practically determines a whole train of microphysical phenomena.

The remaining papers related to heavy-duty contacts: Mr. Baxter (Electrical Research Association) reviewed factors causing wear, and Dr. Wilson (General Electric Co., Ltd.) discussed heavy contactors. R. W. Sillars (Metropolitan-Vickers Electrical Co., Ltd.) dealt with are suppression by associated circuits, T. H. Cook and M. Vaughan (Morgan Crucible Co., Ltd.) with brush contact efficiency, P. F. Soper (Northampton Polytechnic) with sliding contacts, and R. F. Sims (Royal Aircraft Establishment, Farnborough) with brush contacts operating at high altitudes. F. LLEWELLYN JoNes

\section{SUGGESTED REFORM OF THE INDIAN CALENDAR}

$A^{\mathrm{T}}$

$T$ the inaugural meeting of the Indian Astronomical and the Astrophysical Society of India, the president, Prof. M. N. Saha, gave an address on "The Reform of the Indian Calendar", which has recently been published (pp. 26 ; Indian Science News Association, 92 Upper Circular Road, Calcutta). Prof. Saha commenced by- explaining the problems of the calendar and described the different adjustments made by various nations in the past to obviate confusion; but in many cases the adjustments were responsible for raising more problems than they settled. He pointed out that, while the Christian calendar gives the length of the year correctly, it has a number of defects, such as the variation in the length of the months, the fact that the beginning of the month and of the year may fall on any day of the week, resulting in inconvenience to civic and economic activities, the use of the moon for determining certain Christian festivals and holidays, such as Easter, etc., and other inconveniences as well.

Prof. Saha went on to discuss two of the proposals that have been made to reform the Christian calendar 
- the thirteen-month calendar and the twelve-month calendar. In the former there would be thirteen months in the year, each of four weeks, a week being, as now, seven days; but in ordinary years the last day would be called 'year-end day', and in leap years there would be an additional 'year-middle day' at the end of July. In the twelve-month calendar, each quarter contains exactly three months-that is, thirteen weeks or ninety-one days-and the quarters begin on Sunday and end on Saturday. The first month in each quarter has thirty-one days and the other two thirty days each. As in the thirteen-month year there would be a 'year-end day' and, in leap years, an additional 'year-middle day'.

A historical sketch was given in the address of the Indian calendars, of which there have been twentyfive different kinds in use. They are divided into three categories: calendars of purely Indian origin; calendars of foreign origin-Christian, Hedjirah Tarikh-Ilahi; and hybrid calendars. Those who are interested in Indian calendars will find a very full description of them, and in an appendix the proposals for a universal calendar are set forth.

Briefly, Prof. Saha suggests that the year should commence on December 22, the winter solstice day, which will always be a Sunday. This new year's day will also be Christmas day, on which various festivals of other nations will be celebrated. The four quarters will each contain ninety-one days, the months of each quarter consisting of thirty-one, thirty and thirty days, and each quarter will start on a Sunday and end on a Saturday. The first, or winter, quarter will end on March 22, which will then be the thirtieth day of the third month, and vernal equinox will be on the previous day. The second, or spring, quarter will start on March 23 and end on June 21, summer solstice day; and the third, or summer, quarter will begin on June 22 and end on September 20, which will be a Saturday. The fourth, or autumn, quarter will commence on Sunday, September 22 (not on September 21, which can be regarded as an extra day and may be called the 'world day'), and the Monday following September 22 is the autumnal equinox. In the case of a leap year, another day can be interposed just before the winter equinox. This proposed calendar, set out fully in the address, with the months numbered 1 to 12 and the names of the corresponding Indian months.

\section{VELOCITY DISTRIBUTION OF SPORADIC METEORS}

A

PAPER entitled "The Velocity Distribution of Sporadic Meteors", by Mary Almond, J. G. Davies and A. C. B. Lovell ${ }^{1}$, has been published which describes the radio-diffraction technique used at Jodrell Bank Experimental Station of the University of Manchester for measuring the velocities of sporadic meteors. While no doubt exists about the elliptical orbits of shower meteors, certain difficulties of technique-particularly in measurements of velocity-have prevented complete agreement on the orbits of sporadic meteors. The observational work of Prentice and Alcock, and Porter's comprehensive analysis of data on meteors, showed that if hyperbolic meteors existed they were extremely rare, and Whipple's photographic methods support this con- clusion. Since 1947, radio-echo methods for measuring meteor velocities have been in use, and the technique developed by Davies and Ellyett for the observation of the diffraction pattern produced by meteor trails during their formation has been described ${ }^{2}$. The results confirm that shower meteors have velocities below the parabolic limit. The Jodrell Bank paper gives an account of the work on the velocity distribution of the sporadic meteors.

A full description of the apparatus and method of measurement of velocity is followed in the Jodrell Bank paper by tables giving the results of experiments carried out during the autumn mornings of 1948 , using the $4 \cdot 16-\mathrm{m}$. equipment and steerable array, and during the autumn mornings of 1949 and the spring evenings of 1950 , with the $8 \cdot 13-\mathrm{m}$. equipment and fixed array. In the first case, 67 velocities were measured during 230 hours of observation; in the second case, 187 velocity measurements were made during 43.5 hours of observation. In both cases the experiments were concerned with swift meteors emanating from positions in the direction of the apex of the earth's way, and the smooth curves shown in Figs. 1 and 2 of the paper-calculated on the assumption of meteors moving with parabolic velocities and random directions, errors of measurements being superimposed-indicate very close agreement with the observed distribution. The antapex experiments from February 18 until April 29, 1950 , observations being carried out between $15 \mathrm{~h}$. and $20 \mathrm{~h}$., supplied 87 velocities during a total of 213 hours, and a significant difference appeared between the observed and theoretical results. The speeds of the meteors were much smaller than the predicted values, which is attributed to one of two causes or probably to a combination of both. Either the meteors are moving in very elliptical orbits, or their radiants are concentrated towards the plane of the ecliptic; and there is a significant resemblance to the short-period comets, which have elliptical orbits, small inelinations, and nearly always direct motion. In the morning hours the atmosphere is shielded by the earth from meteors with small inclinations and direct motion, but in the early evening hours these can be seen to advantage. In the case of the observations of the meteors during the spring evenings of 1950 , it is significant that only one meteor was observed with velocity between 60 and $70 \mathrm{~km}$. $/ \mathrm{sec}$., whereas in the observations during the morning hours of the previous autumn there were sixty-eight meteors in this range; and it is certain that the high-velocity cut-off in the former case was a real effect. No evidence was obtained from any of the observations that an appreciable number of meteors were moving in hyperbolic orbits.

Estimates of the magnitude-range of the meteors were made by computation of the electron densities in the meteor trails, these densities being converted to visual meteor magnitudes; but the latter does not give very satisfactory results owing to the uncertainty in the theories of the ionization process. It is believed, however, that the major number of measured velocities is around the fifth magnitude. The results show good agreement with those obtained by McKinley and Millman, who used a different radioecho technique with a different selection of the sporadic meteors. In a later paper the results of the experiments made to cover fainter meteors will be discussed.

'Mon. Not. Roy. Astro. Soc., 111, 6 (1951).

- Nature, 161, 596 (1948). 